\title{
Delay in the alternating-offers model of bargaining*
}

\author{
Juan J. Vidal-Puga ${ }^{\dagger}$
}

March 16, 2008

\begin{abstract}
Alternating offers bargaining has been extensively used to model two-sided negotiations. The celebrated model of Rubinstein (1982) has provided a formal justification for equitable payoff division. A typical assumption of these models under risk is that the termination event means a complete and irrevocable breakdown in negotiations. In this paper, the meaning of termination is reinterpreted as the imposition to finish negotiations immediately. Specifically, bargaining terminates when the last offer becomes definitive. While Rubinstein's model predicts an immediate agreement with stationary strategies, we show that the same payoff allocation is attainable under non-stationary strategies. Moreover, the payoffs in delayed equilibria are potentially better for the proposer than those in which agreement is immediately reached.
\end{abstract}

Keywords: breakdown, bargaining, delay.

JEL codes: C72, C78

\footnotetext{
*Two previous versions of this paper, both titled "Reinterpreting the meaning of breakdown", have circulated as an IVIE working paper (WP-AD 2006-22) at the University of Alicante, and as a RGEA working paper (3-07) at the University of Vigo, respectively. A first version, titled "Why does delay happens in real-life bargaining?" has benefited from helpful comments from Clara Ponsatí. Usual disclaimer applies. This research was partially conducted while the author was visiting the Department of Economic Analysis at the University of Alicante. Financial support by the Spanish Ministerio de Ciencia y Tecnología and FEDER through grant SEJ2005-07637-C02-01/ECON and Xunta de Galicia through grants PGIDIT06PXIC300184PN and PGIDT06PXIB362390PR is also gratefully acknowledged.

${ }^{\dagger}$ Research Group in Economic Analysis. Universidade de Vigo. 36200 Vigo (Pontevedra), Spain. E-mail: vidalpuga@uvigo.es
} 


\section{Introduction}

Many economic situations can be modelled as two agents with independent interests who may benefit from cooperation. However, no agreement can be achieved without the approval of both individuals. Assuming the agents are rational, it seems natural to predict that they will cooperate, and the question arises how the benefits from cooperation are bound to be distributed between them.

In his seminal paper, Rubinstein [20] addressed this question. He studied a simple bargaining protocol of alternating offers between two agents who want to share a pie of size 1 . The agents alternatively propose a share of the pie until a proposal is accepted. No prior deadline is imposed on the number of offers that can be made. The driving force of the negotiation is that agents are impatient. The pie shrinks as time passes by. Binmore, Rubinstein and Wolinsky [2] reformulated the model in a way that the driving force is the possibility of breakdown after a rejection, in which case both agents get nothing. In both models, the only subgame perfect (SP) equilibrium has two main properties: First, it is stationary. Second, the advantage of being the first proposer disappears as the cost of delay (Rubinstein [20]) or the probability of breakdown (Binmore et al. [2]) vanishes ${ }^{1}$.

In the classical literature on bargaining under risk, as in [2], termination means a complete and irrevocable breakdown in negotiations, so that no agreement is possible afterwards. The reason why the agents reach such radical dead-end is explained in [17, p. 73] as follows: the players may get fed up as negotiations become protracted, and thus walk away from the negotiation table [...] Another possible cause for the existence of a risk of breakdown is that 'intervention' by a third party results in the disappearance of the 'gains from cooperation' that exists between the two players.

In the first case, it is certainly plausible that an agent gets fed up and wants to stop negotiating immediately. In this case, however, we claim that the rational thing to do is to accept the last offer on the table (as long as it is better than nothing). In the second case, external intervention may become so imminent that agents are forced to finish negotiations immediately. In this case, we assume that even though there is no time for making a new proposal, or doing so is too costly, it is still possible to accept the last offer.

\footnotetext{
${ }^{1}$ The resemblance between the results in [20] and [2] has led to the interpretation of the cost of delay as an exogenous probability of breakdown.
} 
We emphasize that this assumption represents only a possible scenario, to be contrasted with the classical scenario in which the pie disappears so quickly that there is no time to react, and both players obtain zero.

For example, assume a client asks some firm to estimate the performance of a complex project. Due to this complexity, the firm does not have capacity to perform it by itself. The firm needs either to contract new employers, or to renegotiate the labor conditions with the ones that it already has, or to negotiate with another firm. Assume that during the negotiation process between the firms (or firm-employers), the client gets fed up and decides to cancel the offer so that he can hire another firm. In this new situation, the original firm is always able to make a quick offer that would surely be accepted by the client.

Another example: a patent holder offers a new technology to a coalition of firms, who have to negotiate how to divide the returns from the exploitation of the technology. If the negotiations get protracted, the patent holder may decide to offer the technology to a third party. In this new situation, the coalition of firms is always able to present a quick agreement that would be accepted by the patent holder.

We model these situations as follows: Two agents bargain over the share of a pie of size 1 by alternating offers. Each time an offer is rejected, there is a (small) probability $1-\rho$ that the last proposal becomes a 'take-it-or-leave-it' offer (termination). The responder can then reconsider to accept this last offer. There is a discount factor $\delta<1$, so that an agent's utility for a piece of size $u$ at time $t$ is given by $\delta^{t} u$. If the last offer is rejected after termination, the utility is zero for both agents (breakdown).

Many situations can be described in this manner. First, there can be an external authority that may force the agents to terminate negotiations immediately (for example, a superior authority to which they are delegating). Second, there may exist a small probability that the negotiation proceedings become public and one of the parties cannot change his offer in order to keep its credibility. Third, one of the parties may make a mistake with a small probability (following the ideas presented in [21]) and accept an offer that could have been improved had he been more insistent.

Hence, after an offer is made, the proposer may be committed to it in such a way that further renegotiation is not possible. This leads to an endogenous payoff allocation after bargaining termination, as opposed to the standard 
alternating-offers model under risk ${ }^{2}$.

In real-life negotiations, small delays may happen because the agents begin with proposals that are more favorable to them than the ones they actually consider as the most reasonable (cheap talk). The driving force is that there is a small probability that the other party considers the offer as final.

Our model combines both the discounting factor of [20] and the risk of breakdown of [2]. What induces the agents to reach an agreement is their impatience, and not the risk of termination (as opposed to [2], where termination means breakdown). See [17, Section 4.6] for a model that combines discounting and the risk of breakdown.

There exist models in which the probability of breakdown is not constant. Hart [9, Section III] studied a model in which a crunch happens at round $T$, at which point the size of the pie shrinks rapidly. Even though the breakdown can be seen as a radical crunch, in the model presented in this paper it cannot be anticipated. In [3] and [13], the end of the negotiations depends on the offers and concessions previously made by the agents, and hence it cannot be seem as a termination in the sense studied here.

In Section 2 we summarize the results and compare them with those in the existing literature. In Section 3 we formally present the model and characterize some of the payoffs. In Section 4 we study the stationary SP equilibria. In Section 5 we study the non-stationary SP equilibria. In Section 6 we briefly discuss the case when the agents are risk averse. In Section 7 we give some concluding remarks. In the Appendix (Section 8) we prove most of the results.

\section{Summary of the results}

Our results may be summarized as follows: If the agents are infinitely patient (i.e. they do not care when agreement is reached) then their optimal strategy is to permanently ask for the whole pie. As the agents become more impatient, delay may occur, but an agent eventually presents a reasonable offer and agreement may be reached without termination. If agents are impatient

\footnotetext{
${ }^{2} \mathrm{~A}$ recent model with complete information that makes endogenous the level of surplus destruction after the deadline is [16]. In their model, however, the deadline is also endogenously imposed.
} 
enough, we recover Rubinstein's result of a unique stationary SP equilibrium with immediate agreement.

Our model explains delay maintaining the original assumptions of $\mathrm{Ru}$ binstein's, i.e. unbounded rationality, complete information and no outside options, as well as the most tractable concept of subgame perfect equilibrium. See [6] for a recent work explaining delay in a model with incomplete information. Delay due to outside options can be found in [15] and references therein $^{3}$. Delay due to externalities can be found in [11]. Delay may also arise when the agents can destroy part of the pie $([1,14])$ or are committed not to accept poorer proposals than those already rejected ([7]).

Our model generalizes [20] in the sense that both models coincide when $\rho=1$. However, our model can easily be generalized so as to allow both kinds of termination: the original breakdown of [2] with no possibility of agreement, and our termination with a last 'take-it-or-leave-it' offer.

For arbitrary values of $\rho$ and $\delta$, we can distinguish three regions in the set $\{(\rho, \delta)\}_{\rho, \delta \in] 0,1[}$. A first region $I A$ where "Immediate Agreement" is reached, i.e. the responder agrees on the offer at time 0 (like in [20]). A second region $D A$ ("Delayed Agreement") where agreement is reached, but with a possible delay. Finally, a third region $P D$ ("Perpetual Disagreement") where termination always occurs. The three regions are represented in Figure 1.

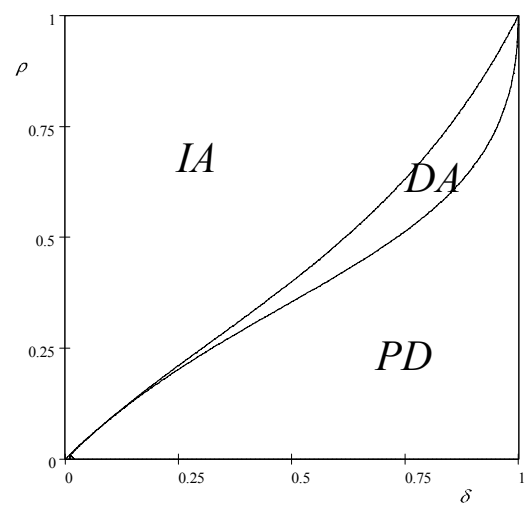

Figure 1: Regions $I A, D A$ and $P D$.

We are interested in studying the agreements that arise when $\rho$ and $\delta$ are close to 1 . A value of $\delta$ close to 1 can be interpreted as either that

\footnotetext{
${ }^{3}$ Outside options may cancel out the effect of incomplete information, as shown in [4].
} 
agents are patient (see [18, p. 52]) or that the interval between offers and counteroffers is short. A value of $\rho$ close to 1 means that the probability of termination is small. In particular, these assumptions arise when the probability of termination (and discounting) depend on time, but the delay $\Delta$ between offers and counteroffers goes to zero. Namely, we can write $\rho=e^{-r \Delta}$ and $\delta=e^{-s \Delta}$ for some $r>0$ and $s>0$. Then, the $P D$ condition is never satisfied for $\Delta$ small enough $^{4}$. Thus, when $\rho$ and $\delta$ are close to $1, P D$ vanishes and the only significant regions are those in which the agents make reasonable offers.

The SP equilibrium payoffs for the proposer as a function of $\delta$ when $\rho=0.5$ are depicted in Figure 2. The figures for other values of $\rho$ are similar. In $I A$ there exists a unique SP equilibrium payoff allocation $\left(\frac{1}{1+\delta}, \frac{\delta}{1+\delta}\right)$ which coincides with Rubinstein's. Moreover, SP equilibria are stationary and efficient (Theorem 1). The dotted line in Figure 2 represents the security payoff $\frac{1-\rho}{1-\rho^{2} \delta^{2}}$, which corresponds to the strategy of permanently asking for the whole pie, and waiting for termination to occur (Remark 1).

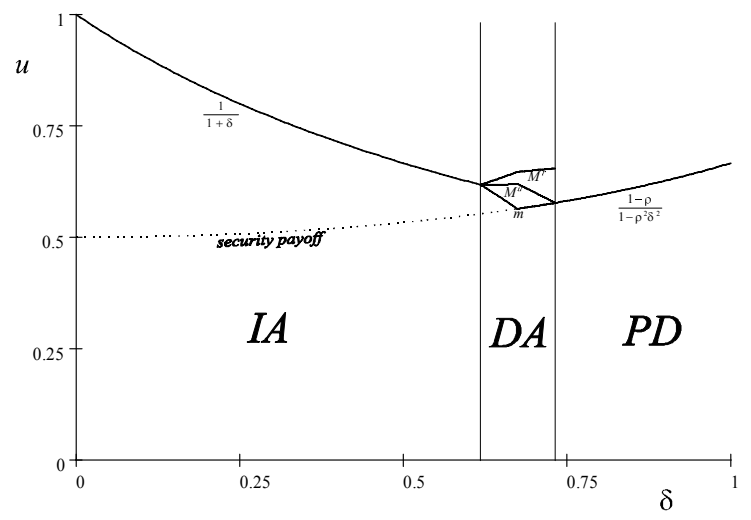

Figure 2: The SP equilibrium payoffs for the proposer as a function of $\delta$ when $\rho=0.5$. The dotted line represents the security level payoff.

In $P D$, there does not exist any $\mathrm{SP}$ equilibrium with immediate agreement, and thus the dotted line in Figure 2 coincides with the unique SP equilibrium payoff (Proposition 1).

In $D A$, there are no stationary SP equilibria, and there exists a continuum of SP equilibrium payoffs (Theorem 2). Remarkably, Rubinstein's alloca-

\footnotetext{
${ }^{4}$ As $\Delta$ aproaches zero, we fall into $I A$ for $2 s>r$; and into $D A$ for $2 s \leq r$.
} 
tion $\left(\frac{1}{1+\delta}, \frac{\delta}{1+\delta}\right)$ is always an attainable outcome in $D A$ under non-stationary strategies for any value of $\rho$ (Corollary 1). Hence, even though Rubinstein's stationary equilibrium is not robust to reinterpretations of the meaning of termination, the allocation $\left(\frac{1}{1+\delta}, \frac{\delta}{1+\delta}\right)$ is. An immediate consequence is that the classical assumption of the meaning of termination as breakdown can be made without loss of generality, as long the final payoff allocation is concerned.

Another remarkable feature in $D A$ is that, in general, the payoffs attainable when agreement is immediate (below $M^{a}$ in Figure 2) are potentially worse for the proposer than those attainable when agreement is delayed (below $M^{r}$ in Figure 2). The interpretation of this phenomenon is as follows: The most unfavorable SP equilibria for the proposer are those in which her initial proposal is accepted. Hence, in general it is beneficial to begin with an unacceptable offer. Do bargainers behave in this manner in real life negotiations? It is obvious that they do. For example, in the Spanish house market, the consultants Re/Max and Look\&Find have recently pointed out that sellers put a high price planning to decrease it during the bargaining. Claiming high prices gives somehow a favorable status quo in case negotiations should finish immediately.

It should be stressed that we characterize all the SP equilibria payoffs, and not only the stationary ones. There are no stationary SP equilibria in $D A$, only non-stationary ones. The result is also true for the other direction: if there are non-stationary SP equilibria, then there are no stationary ones. In $I A$, the only stationary SP equilibrium is efficient (i.e. agreement is reached immediately). In $P D$, the only stationary SP equilibrium is inefficient (agreement is only reached after termination).

\section{The model}

Two agents, $A$ and $B$, bargain over a pie of size 1 following a protocol of alternating offers: At time $t=0,2,4, \ldots$, agent $A$ is the proposer. At time $t=1,3,5, \ldots$, agent $B$ is the proposer. At time $t=0, A$ proposes a division $x=\left(x_{A}, x_{B}\right)$, where $x_{A}+x_{B} \leq 1$. $B$ should then accept or reject the offer. If he accepts, the game finishes with this division at time $t=0$. If he rejects, then with probability $\rho$, the process is repeated at time $t=1$, with $B$ being the proposer. With probability $1-\rho$, the proposal made by $A$ becomes final (termination), and $B$ should decide if he accepts or rejects it. If he accepts, 
the game finishes with this share at time $t=0$. If he rejects, the game finishes in disagreement and each agent receives zero (see Figure 3). Each time an offer is rejected, the value of the pie decreases by a factor of $\delta \in[0,1[$ for each agent. This means that a share of $x_{i}$ of the cake at time $t$ yields a utility of $\delta^{t} x_{i}$ for agent $i$.

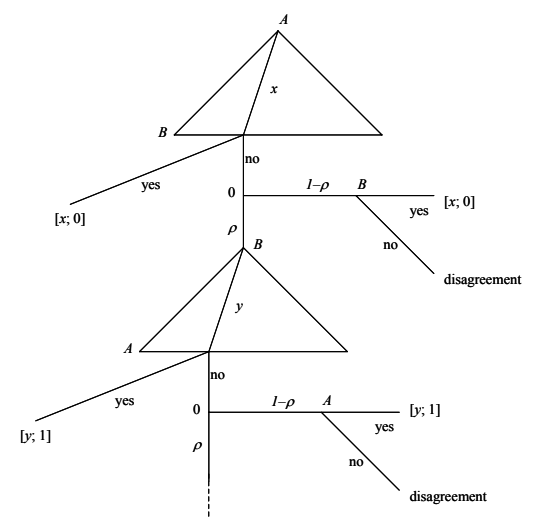

Figure 3: The first two periods of the bargaining model.

The factor $1-\rho$ can be considered as some kind of external factor which represents the belief that the proposal on the table will become a 'take-it-orleave-it' offer. On the other hand, the discount factor $\delta$ may be an internal factor that represents impatience.

For simplicity, we assume that agents propose admissible shares of the pie (i.e. $x_{i} \in[0,1]$ for $i \in\{A, B\}$ besides $\left.x_{A}+x_{B} \leq 1\right)$. By termination we mean that the last offer is made final (and not necessarily that each agent receives zero). We say that bargaining finishes in agreement when the agents follow strategies in which an offer is accepted without termination.

The structure of the game is stationary, and so all the subgames that begin when agent $i$ makes an offer are equivalent. Let $U$ be the set of possible SP equilibrium payoffs for the proposer in these subgames (by symmetry, this set is the same for both agents). If $U$ is nonempty, we denote the supremum of $U$ as $M$, and the infimum of $U$ as $m$.

There are two kinds of SP equilibria. Let $U^{a}$ be the set of payoffs for $A$ in the equilibria in which the first offer is accepted. Let $U^{r}$ be the set of payoffs for $A$ in the equilibria in which the first offer is rejected. 
Remark 1 In this model, A can assure herself a positive expected final payoff by always offering $(1-\varepsilon, \varepsilon)$. If her offer is rejected but termination occurs, perfectness of the equilibrium will imply that $B$ accepts. Since this is true for any $\varepsilon>0$, we assume without loss of generality that an agent always accepts a payoff of at least 0 when termination occurs. Hence, the SP equilibrium payoff ${ }^{5}$ for the proposer is at least

$$
\sum_{t=0}^{\infty}(\rho \delta)^{2 t}(1-\rho)=\frac{1-\rho}{1-\rho^{2} \delta^{2}}
$$

This is the final payoff for the proposer when both agents always ask for the whole pie. It represents the security level payoff for the proposer. Hence, if $U \neq \emptyset$,

$$
\frac{1-\rho}{1-\rho^{2} \delta^{2}} \leq m
$$

Intuitively, it seems clear that if the agents are sufficiently patient $(\delta$ large) or the risk of breakdown is sufficiently large ( $\rho$ small), then they will keep asking for the whole pie until a proposal is made final (termination). We will see that the set of pairs $(\rho, \delta)$ where this happens is

$$
P D:=\left\{(\rho, \delta): \delta(1-\rho)>\rho\left(1-\rho \delta^{2}\right)\right\}
$$

where PD stands for "Perpetual Disagreement".

Proposition 1 If $(\rho, \delta) \in P D$, then there exists a unique SP equilibrium. In this equilibrium, the proposer always claims the whole pie, and the responder rejects when this proposal is not final. Moreover,

$$
U=U^{r}=\left\{\frac{1-\rho}{1-\rho^{2} \delta^{2}}\right\} .
$$

Proof. Assume we are in a SP equilibrium and the proposer, say $A$, offers $\left(x_{A}, x_{B}\right)$ and $B$ accepts. By rejecting, $B$ can ensure himself a final payoff of at least $\rho \delta m+(1-\rho) x_{B}$. Thus, $\rho \delta m+(1-\rho) x_{B} \leq x_{B}$, i.e. $\delta m \leq x_{B}$. Since $x_{A}+x_{B} \leq 1$, we have $x_{A} \leq 1-\delta m$. On the other hand, $A$ can assure herself a payoff of at least $m$. Thus, $m \leq x_{A}$. Under (1),

$$
\begin{aligned}
m & \leq x_{A} \leq 1-\delta m \\
\frac{1-\rho}{1-\rho^{2} \delta^{2}} & \leq x_{A} \leq 1-\delta \frac{1-\rho}{1-\rho^{2} \delta^{2}}
\end{aligned}
$$

\footnotetext{
${ }^{5}$ From now on, we omit the term 'expected' when referring to final payoffs.
} 
which implies $1-\rho \leq 1-\rho^{2} \delta^{2}-\delta(1-\rho)$. Thus $\delta(1-\rho) \leq \rho\left(1-\rho \delta^{2}\right)$ and hence $(\rho, \delta) \notin P D$. This means that in $P D$ there cannot be an accepted proposal in SP equilibrium. From this, it is clear that the only optimal strategy is to ask for the whole pie. In particular, consider the following strategy for $A$ : if she is the proposer, she offers $(1,0)$; if she is the responder and the offer is $\left(x_{A}, x_{B}\right)$, she accepts iff $x_{A}>\frac{\delta(1-\rho)}{1-\rho^{2} \delta^{2}}$. In case of termination, she accepts any offer. $B$ follows the same strategy.

These strategies constitute a SP equilibrium when $(\rho, \delta) \in P D$.

If $A$ deviates when she is the proposer, she receives at most $1-\frac{\delta(1-\rho)}{1-\rho^{2} \delta^{2}}$, an amount that is strictly less than $\frac{1-\rho}{1-\rho^{2} \delta^{2}}$ in $P D$.

If $A$ deviates when she is the responder and accepts $x_{A} \leq \frac{\delta(1-\rho)}{1-\rho^{2} \delta^{2}}$, she will receive no more than

$$
\rho \delta \frac{1-\rho}{1-\rho^{2} \delta^{2}}+(1-\rho) x_{A}
$$

which is the payoff she would receive by rejecting. Finally, if $A$ deviates when she is the responder and rejects $x_{A}>\frac{\delta(1-\rho)}{1-\rho^{2} \delta^{2}}$, she will get (2), which is strictly less than $x_{A}$.

The set $P D$ is represented in Figure 1.

\section{$4 \quad$ Stationary SP equilibria}

We study the SP equilibria which satisfy the following properties:

Efficiency Whenever a proposer makes an offer, this proposal is accepted by the responder. Moreover, the proposals always satisfy $x_{A}+x_{B}=1$.

Stationarity The proposer always makes the same offer.

Given stationarity, we denote the offer made by agent $i$ in SP equilibrium as $x_{i}^{*}$. Given efficiency, when $A$ proposes $\left(x_{A}^{*}, 1-x_{A}^{*}\right), B$ accepts. By perfectness, the offer made by $A$ should leave $B$ indifferent to accepting or rejecting. If $B$ rejects and there is no termination (probability $\rho$ ), $B$ will propose $\left(1-x_{B}^{*}, x_{B}^{*}\right)$ and this proposal is accepted by $A$ (by efficiency). Thus, $1-x_{A}^{*}=\rho \delta x_{B}^{*}+(1-\rho)\left(1-x_{A}^{*}\right)$. Analogously, $1-x_{B}^{*}=$ $\rho \delta x_{A}^{*}+(1-\rho)\left(1-x_{B}^{*}\right)$. 
We obtain the unique solution

$$
x_{A}^{*}=x_{B}^{*}=\frac{1}{1+\delta} .
$$

Thus, any SP equilibrium payoff satisfying efficiency and stationarity is characterized by this offer. Notice that this payoff for the proposer coincides with the one proposed by Rubinstein [20]. Such a strategy profile is a SP equilibrium in the following region:

$$
I A:=\left\{(\rho, \delta): \rho\left(1-\delta^{2}\right)>\delta(1-\rho)\right\}
$$

where IA stands for "Immediate Agreement".

Theorem 1 a) There exists an efficient and stationary SP equilibrium if and only if $\delta(1-\rho) \leq \rho\left(1-\delta^{2}\right)$.

b) if $\rho\left(1-\delta^{2}\right)>\delta(1-\rho)$, then the above SP equilibrium is unique; and

c) if $\delta(1-\rho)=\rho\left(1-\delta^{2}\right)$, then there exists one inefficient SP equilibrium. In this SP equilibrium, $A$ asks for the whole pie in the first round, $B$ rejects and proposes $\left(\frac{\delta}{1+\delta}, \frac{1}{1+\delta}\right)$ in the second round, and $A$ accepts.

Moreover, $\delta(1-\rho) \leq \rho\left(1-\delta^{2}\right)$ implies $U=U^{a}=\left\{\frac{1}{1+\delta}\right\}$.

Proof. See Appendix.

We represent the set $I A$ in Figure 1. The set includes all the pairs $(\rho, \delta)$ with $\rho=1$, where this model coincides with Rubinstein's [20].

\section{Non-stationary SP equilibria}

Since we have characterized the SP equilibria in $I A$ and $P D$, we focus our attention on the remaining values of $(\rho, \delta)$. We define

$$
D A:=\{(\rho, \delta):(\rho, \delta) \notin I A \cup P D\}
$$

where $D A$ stands for "Delayed Agreement".

Let $\widetilde{m}:=\max \left\{\frac{(1-\delta)(1+\rho \delta)}{1-\rho \delta^{2}}, \frac{1-\rho}{1-\rho^{2} \delta^{2}}\right\}, \widetilde{M^{r}}:=\rho \delta(1-\widetilde{m})+1-\rho$; and $\widetilde{M^{a}}:=$ $1-\delta \widetilde{m}$.

Theorem 2 If $(\rho, \delta) \in D A$, then there exist non-stationary SP equilibria. Furthermore, the set of SP equilibrium payoffs for the proposer is given by $U=U^{r}=\left[\widetilde{m}, \widetilde{M^{r}}\right]$ and $U^{a}=\left[\widetilde{m}, \widetilde{M^{a}}\right]$. 
Proof. See Appendix.

These intervals are represented in Figure 2 for $\rho=0.5$. Notice that when $(\rho, \delta)$ belongs to the interior of $D A$, we have $\widetilde{M^{r}}>\widetilde{M^{a}}$.

The strategy profiles which yield these intervals are described in Table I. We use the language of automata (see [18, p. 39-40]). Acceptance applies when the offer is not final. If the offer is final, the responder always accepts (as explained in Remark 1). The strategies are the most favorable to $A$ in column Favor $A$; the strategies are the most favorable to $B$ in column Favor $B$; the initial offer is accepted in column Agreement when $\widetilde{M^{a}}>u$; and the initial offer is rejected in column Delay.

TABLE I: SP equilibrium strategies in $D A$

\begin{tabular}{|c|c|c|c|c|}
\hline & Favor $A$ & Favor $B$ & Agreement & Delay \\
\hline$A$ offers & $(1,0)$ & $(\widetilde{m}, 1-\widetilde{m})$ & $(u, 1-u)$ & $\left(\frac{u-\rho \delta(1-\widetilde{m})}{1-\rho}, 0\right)$ \\
\hline$A$ accepts & $x_{A} \geq 1-\widetilde{m}$ & $x_{A}>\delta \widetilde{m}$ & - & - \\
\hline$B$ offers & $(1-\widetilde{m}, \widetilde{m})$ & $(0,1)$ & - & - \\
\hline$B$ accepts & $x_{B}>\delta \widetilde{m}$ & $x_{B} \geq 1-\widetilde{m}$ & - & - \\
\hline Transitions & $\begin{array}{l}\text { if } A \text { deviates, } \\
\text { go to Favor } B\end{array}$ & $\begin{array}{l}\text { if } B \text { deviates, } \\
\text { go to Favor } A\end{array}$ & $\begin{array}{l}\text { if } A \text { deviates, } \\
\text { Otherwise, }\end{array}$ & $\begin{array}{l}\text { go to Favor } B \\
\text { go to Favor } A \text {. }\end{array}$ \\
\hline
\end{tabular}

The following corollary is an important consequence of Theorem 2.

Corollary 1 Given $(\rho, \delta) \in D A, \frac{1}{1+\delta} \in U^{a}$.

Proof. From Theorem 2, it is enough to prove that $\widetilde{m} \leq \frac{1}{1+\delta} \leq \widetilde{M^{a}}$.

Assume first $\widetilde{m}=\frac{(1-\delta)(1+\rho \delta)}{1-\rho \delta^{2}}$. We have to prove $\frac{(1-\delta)(1+\rho \delta)}{1-\rho \delta^{2}} \leq \frac{1}{1+\delta} \leq$ $1-\delta \frac{(1-\delta)(1+\rho \delta)}{1-\rho \delta^{2}}$. The first inequality is equivalent to $\rho \delta\left(1-\delta^{2}\right) \leq \delta^{2}(1-\rho)$, which holds because $(\delta, \rho) \notin I A$. The second inequality is equivalent to $\rho \delta^{2}\left(1-\delta^{2}\right) \leq \delta^{3}(1-\rho)$, which holds because $(\delta, \rho) \notin I A$.

Assume now $\widetilde{m}=\frac{1-\rho}{1-\rho^{2} \delta^{2}}$. We have to prove $\frac{1-\rho}{1-\rho^{2} \delta^{2}} \leq \frac{1}{1+\delta} \leq 1-\delta \frac{1-\rho}{1-\rho^{2} \delta^{2}}$. The first inequality is equivalent to $\delta(1-\rho) \leq \rho\left(1-\rho \delta^{2}\right)$, which holds because $(\delta, \rho) \notin P D$. The second inequality is equivalent to $\delta^{2}(1-\rho) \leq$ $\rho \delta\left(1-\rho \delta^{2}\right)$, which holds because $(\delta, \rho) \notin P D$. 


\section{Risk aversion}

In the previous sections we have assumed that the agents are risk-neutral. Hence, a natural question is how risk aversion would affect our results. In the original Rubinstein's paper there is no randomness and hence no need to discuss risk attitudes. However, in our model risk aversion is not innocuous because the agents have to make comparisons between lotteries.

Without going into formal details, we can still conjecture how risk aversion affects our results. We study the frontier line between $I A$ and $D A$, denoted as $\partial A:=\left\{(\rho, \delta): \delta(1-\rho)=\rho\left(1-\delta^{2}\right)\right\}$, and between $D A$ and $P D$, denoted as $\partial D:=\left\{(\rho, \delta): \delta(1-\rho)=\rho\left(1-\rho \delta^{2}\right)\right\}$.

By Theorem 1, when $(\rho, \delta) \in \partial A$ and the agents are risk-neutral, there exist exactly two possibilities for SP equilibria, namely:

- $A$ offers $\left(\frac{1}{1+\delta}, \frac{\delta}{1+\delta}\right)$ and $B$ accepts.

- $A$ offers $(1,0)$ and $B$ rejects. If there is no termination, $B$ offers $\left(\frac{\delta}{1+\delta}, \frac{1}{1+\delta}\right)$ and $A$ accepts.

In both cases, $A$ gets a final (expected) payoff of $\frac{1}{1+\delta}$. In the first case, $A$ gets $\frac{1}{1+\delta}$. In the second case, $A$ gets either 1 (with probability $1-\rho$ ) or $\frac{\delta^{2}}{1+\delta}$ (with probability $\rho$ ).

However, under risk aversion $A$ would strictly prefer to get $\frac{1}{1+\delta}$. Hence, one may expect that the set $I A$ "advances" whilst the set $D A$ "retreats". This implies that immediate agreement is more likely under risk aversion.

A similar argument can be followed for $\partial D$. When the agents are riskneutral, the strategy of asking for the whole pie (waiting for breakdown to occur) is a possible SP equilibrium in both $P D$ and $\partial D$. However, under risk aversion this "security" strategy is less attractive, and hence one may expect that the set $D A$ "advances" whilst the set $P D$ "retreats". This implies that agreement is more likely under risk aversion.

\section{Concluding remarks}

The importance of the alternating-offers model rests on two important features. First, it comprises most of the ideas that one may expect to find in a real process of negotiation (see [10] for a nice discussion). For example, time 
is valuable, and still the agents perceive that they always have the choice to make a new offer and continue the negotiation under more or less the same circumstances. Second, it answers the question of what is a reasonable payoff division that the agents are bound to agree. For example, Corominas-Bosch [5] avoids the details of the bargaining process between pairs in a network by simply assigning the division $\left(\frac{1}{1+\delta}, \frac{\delta}{1+\delta}\right)$ in two-sided negotiations.

On the other hand, stationary strategies in general, and immediate agreements in particular, are somehow problematic features. They do not seem to have a counterpart in real-life negotiations. Stationary strategies imply that each agent should not take into account previous offers and rejections. Immediate agreement rules out the possibility of small and harmless delays that seem very willing to happen in real-life negotiations.

This paper maintains the main features of the classical alternating offers model. In particular, it explains delay maintaining the original assumptions of Rubinstein's, i.e. unbounded rationality, complete information and no outside options, as well as the most tractable concept of subgame perfect equilibrium.

It is worth to note that for some parameter values there exist equilibria in which the players begin by making unacceptable offers. In the classical work of Harsanyi-Zeuthen [23, 8] and Raiffa [19] (see [12, Ch. 6.7]), the players are assumed to start by making unacceptable proposals, and then go though a sequence of concessions. The Harsanyi-Zeuthen formulation leads to the Nash bargaining solution, whilst the Raiffa process leads to a different point. Even though there exist non-cooperative bargaining games that lead to the Nash (as for example [2]) or Raiffa (see [22]) solutions, the equilibria of these games do not have the property that the players start by making unacceptable demands.

\section{Appendix}

Lemma $11-\delta M \leq m$.

Proof. Clearly, $B$ would not reject any offer $\left(x_{A}, x_{B}\right)$ with $x_{B}$ satisfying $x_{B}>\rho \delta M+(1-\rho) x_{B}$, i.e. $x_{B}>\delta M$, because $\rho \delta M+(1-\rho) x_{B}$ would be his maximum payoff after rejection. Thus, there does not exist $u \in U$ such that $1-\delta M>u$. Hence, $1-\delta M \leq m$. 


\subsection{Proof of Theorem 1}

a) Let $\overline{I A}:=\left\{(\rho, \delta): \delta(1-\rho) \leq \rho\left(1-\delta^{2}\right)\right\}$ denote the closure of $I A$. We have previously proved that any stationary and efficient SP equilibrium is characterized by (3). We now check that the strategy profile in which both agents "offer $\frac{1}{1+\delta}$ and accept at least $\frac{\delta}{1+\delta}$ " constitutes a SP equilibrium in $\overline{I A}$.

Assume the proposer $A$ deviates and makes an unacceptable proposal $\left(x_{A}, x_{B}\right)$. Then, $B$ rejects and $A$ 's final payoff is $\rho \delta \frac{\delta}{1+\delta}+(1-\rho) x_{A}$, which should be no more than $\frac{1}{1+\delta}$, i.e. $\rho \delta \frac{\delta}{1+\delta}+(1-\rho) x_{A} \leq \frac{1}{1+\delta}$. Since $x_{A} \leq 1$, it is enough to prove that $\rho \delta \frac{\delta}{1+\delta}+1-\rho \leq \frac{1}{1+\delta}$, which holds because $(\rho, \delta) \in \overline{I A}$.

Assume the responder $B$ receives an offer $\left(x_{A}, x_{B}\right)$. By rejecting, he gets in expected terms $\rho \delta \frac{1}{1+\delta}+(1-\rho) x_{B}$. Thus, it is optimal to accept iff $\rho \delta \frac{1}{1+\delta}+(1-\rho) x_{B} \leq x_{B}$, i.e. $\frac{\delta}{1+\delta} \leq x_{B}$. Hence the strategy for $B$ is optimal. This proves that there exists a stationary SP equilibrium when $(\rho, \delta) \in \overline{I A}$.

Assume now $(\rho, \delta) \notin \overline{I A}$. Assume the proposer $A$ deviates and proposes $(1,0)$. Her final payoff is $\rho \delta \frac{\delta}{1+\delta}+1-\rho$. Since $(\rho, \delta) \notin \overline{I A}$, this payoff is greater than $\frac{1}{1+\delta}$. Hence, $A$ improves. This shows that there is no efficient and stationary SP equilibria when $(\rho, \delta) \notin \overline{I A}$.

We now prove that $U=\left\{\frac{1}{1+\delta}\right\}$. Since the above strategies constitute a SP equilibrium in $\overline{I A}$, we know that $U \neq \emptyset$ in $\overline{I A}$.

We will prove that $M=m=\frac{1}{1+\delta}$. We proceed with a series of Claims.

Claim $1 M \leq 1-\delta m$.

Proof. Let $\left(x_{A}, x_{B}\right)$ be $A$ 's proposal. In a SP equilibrium, $B$ rejects any offer such that $\rho \delta m+(1-\rho) x_{B}>x_{B}$, i.e. such that $\delta m>x_{B}$. Since $x_{A}+x_{B} \leq 1$, this means that $B$ rejects any offer such that $x_{A}>1-\delta m$.

There exist two types of SP equilibria: (I) Equilibria in which $B$ accepts $A$ 's initial offer, and (II) equilibria in which $B$ rejects $A$ 's initial offer.

In equilibria of type (I), it is clear that $x_{A} \leq 1-\delta m$. In equilibria of type (II), let $u$ be $A$ 's final payoff and let $v$ be $B$ 's final payoff. If $B$ becomes the proposer (this occurs with probability $\rho$ ), $B$ gets $u^{\prime} \in U$ and $A$ gets $v^{\prime}$. Since $u^{\prime}+v^{\prime} \leq 1$, we deduce that $v^{\prime} \leq 1-u^{\prime} \leq 1-m$. Moreover, $x_{A} \leq 1$. Thus, $u=\rho \delta v^{\prime}+(1-\rho) x_{A} \leq \rho \delta(1-m)+1-\rho$. In summary, $u \leq \max \{1-\delta m, \rho \delta(1-m)+1-\rho\}$. We will prove that this maximum is $1-\delta m$ and thus $M \leq 1-\delta m$. We want to prove that $\rho \delta(1-m)+1-\rho \leq$ 
$1-\delta m$, i.e. $\delta(1-\rho) m \leq \rho(1-\delta)$. Since $m \leq \frac{1}{1+\delta}$ in $\overline{I A}$, it is enough to prove that $\delta \frac{1-\rho}{1+\delta} \leq \rho(1-\delta)$, which holds because $(\rho, \delta) \in \overline{I A}$.

Claim $2 M \leq \frac{1}{1+\delta}$.

Proof. Under Claim 1 and Lemma $1, M \leq 1-\delta m \leq 1-\delta(1-\delta M)$, and thus $\left(1-\delta^{2}\right) M \leq 1-\delta$, hence $M \leq \frac{1}{1+\delta}$.

Claim $31-\delta u \in U$ for all $u \in U$.

Proof. Let $u \in U$, and let $v$ be $A$ 's final payoff in a subgame in which $B$ is the proposer and he gets $u$. Hence, $v \leq 1-u$. We consider the following strategies: $A$ proposes $(1-\delta u, \delta u)$ and $B$ accepts an offer $\left(x_{A}, x_{B}\right)$ iff $\delta u \leq x_{B}$. Thereafter, agents play the strategies that yield $u$ to $B$ and $v$ to $A$. We will check that these strategies constitute a SP equilibrium. It is clear that these strategies are an SP equilibrium after the first offer has been rejected.

If $B$ rejects a proposal $\left(x_{A}, x_{B}\right)$ with $\delta u \leq x_{B}$, he gets $\rho \delta u+(1-\rho) x_{B} \leq$ $\rho x_{B}+(1-\rho) x_{B}=x_{B}$, and his payoff does not increase. Analogously, $B$ 's payoff does not increase by accepting a proposal $\left(x_{A}, x_{B}\right)$ with $\delta u>x_{B}$.

If $A$ deviates and proposes $x_{A}>1-\delta u$, he gets $\rho \delta v+(1-\rho) x_{A}$. We need to prove that $A$ does not improve, i.e. $\rho \delta v+(1-\rho) x_{A} \leq 1-\delta u$. Since $v \leq 1-u$ and $x_{A} \leq 1$, it is enough to prove that $\rho \delta(1-u)+1-\rho \leq 1-\delta u$, i.e. $u \leq \frac{\rho(1-\delta)}{\delta(1-\rho)}$. Since $u \leq M$, under Claim 2 it is enough to prove that $\frac{1}{1+\delta} \leq \frac{\rho(1-\delta)}{\delta(1-\rho)}$, which holds because $(\rho, \delta) \in \overline{I A}$.

Claim $4 m \leq 1-\delta M$ and $1-\delta m \leq M$.

Proof. Assume $m>1-\delta M$. Thus, there exists $u \in U$ such that $m>1-\delta u$. But by Claim 3 this is not possible.

Assume $1-\delta m>M$. Thus, there exists $u \in U$ such that $1-\delta u>M$. But this is not possible under Claim 3.

From Lemma 1, Claim 1 and Claim 4, we deduce that $m=1-\delta M$ and $M=1-\delta m$, which yield a unique solution $M=m=\frac{1}{1+\delta}$. This proves that $U=\left\{\frac{1}{1+\delta}\right\}$ and completes the proof of part a).

b) Assume $\rho\left(1-\delta^{2}\right)>\delta(1-\rho)$. It is enough to prove that all SP equilibria are efficient. Assume, on the contrary, that there exists a SP 
equilibrium where $A$ proposes $\left(x_{A}, x_{B}\right)$ and $B$ rejects. Let $v$ be $A$ 's payoff in the subgame which begins when $B$ is the proposer. Then, $A$ 's final payoff is $\rho \delta v+(1-\rho) x_{A} \leq \rho \delta \frac{\delta}{1+\delta}+1-\rho$. Since $A$ 's final payoff is $\frac{1}{1+\delta}$, we deduce that $\frac{1}{1+\delta} \leq \rho \delta \frac{\delta}{1+\delta}+1-\rho$ which is not possible when $\rho\left(1-\delta^{2}\right)>\delta(1-\rho)$.

c) Assume $\delta(1-\rho)=\rho\left(1-\delta^{2}\right)$. Assume $A$ proposes $\left(x_{A}, x_{B}\right)$ and $B$ rejects. Let $v$ be $A$ 's payoff in the subgame which begins when $B$ is the proposer. By an analogous argument as in the proof of part b), we prove that $\rho \delta v+(1-\rho) x_{A}=\rho \delta \frac{\delta}{1+\delta}+1-\rho$ and thus $v=\frac{\delta}{1+\delta}$ and $x_{A}=1$. This implies that $A$ asks for the whole pie in the first round (because $x_{A}=1$ ) and that the second round is efficient (because $v=\frac{\delta}{1+\delta}$ ).

\subsection{Proof of Theorem 2}

Let $u \in\left[\widetilde{m}, \widetilde{M^{r}}\right]$. We describe the strategies that yield $u$ as final payoff for the proposer in Table I. Acceptance applies when the offer is not final. If the offer is final, the responder always accepts.

We will prove that the strategies are the most favorable to $A$ (resp. $B$ ) in Favor A (resp. Favor B); the initial offer is accepted in Agreement when $\widetilde{M^{a}}>u$; and the initial offer is rejected in Delay.

In order to check that these strategies are well-defined, we prove that $\frac{u-\rho \delta(1-\widetilde{m})}{1-\rho} \leq 1$, or equivalently, $\widetilde{m} \leq \frac{1-\rho+\rho \delta-u}{\rho \delta}$. Since $u \leq \widetilde{M^{r}}$, it is enough to prove that $\widetilde{m} \leq \frac{1-\rho+\rho \delta \delta \widetilde{M^{r}}}{\rho \delta}$, which holds because $\widetilde{M^{r}}=\rho \delta(1-\widetilde{m})+1-\rho$.

We now check that these strategies are a SP equilibrium.

Favor A and Favor B: Assume the state is Favor $A$. The proof for Favor $B$ is analogous (note that both states are symmetric). If the proposer is $B$, then his offer is accepted and the final payoff is $1-\widetilde{m}$ for $A$ and $\widetilde{m}$ for $B$. If the proposer is $A$, then her offer is rejected and the final payoffs are $\rho \delta(1-\widetilde{m})+1-\rho=\widetilde{M^{r}}$ for $A$ and $\rho \delta \widetilde{m}$ for $B$.

We prove that these strategies are optimal.

A's strategy is optimal when she is the responder. If $A$ rejects $\left(x_{A}, x_{B}\right)$ with $1-\widetilde{m} \leq x_{A}$, we will go to Favor $B, B$ only accepts $x_{B} \geq 1-\widetilde{m}$ and proposes $(0,1)$. Thus, $A$ 's final payoff is at most $\rho \delta \widetilde{m}+(1-\rho) x_{A}$ (if $A$ offers $x_{B} \geq 1-\widetilde{m}$ afterwards) or $\frac{\rho \delta(1-\rho)}{1-\rho^{2} \delta^{2}}+(1-\rho) x_{A}$ (if $A$ permanently makes unacceptable offers). We have to prove that $x_{A}$ is not less than these payoffs. 
1. First, inequality $\rho \delta \widetilde{m}+(1-\rho) x_{A} \leq x_{A}$ is equivalent to $\widetilde{m} \leq \frac{x_{A}}{\delta}$. Since $1-\widetilde{m} \leq x_{A}$, it is enough to prove $\widetilde{m} \leq \frac{1-\widetilde{m}}{\delta}$, i.e. $\widetilde{m} \leq \frac{1}{1+\delta}$.

(a) Assume first $\widetilde{m}=\frac{(1-\delta)(1+\rho \delta)}{1-\rho \delta^{2}}$. We have to prove $\frac{(1-\delta)(1+\rho \delta)}{1-\rho \delta^{2}} \leq \frac{1}{1+\delta}$, which holds because $(\rho, \delta) \notin I A$.

(b) Assume now $\widetilde{m}=\frac{1-\rho}{1-\rho^{2} \delta^{2}}$. We have to prove $\frac{1-\rho}{1-\rho^{2} \delta^{2}} \leq \frac{1}{1+\delta}$, which holds because $(\rho, \delta) \notin P D$.

2. Second, inequality $\frac{\rho \delta(1-\rho)}{1-\rho^{2} \delta^{2}}+(1-\rho) x_{A} \leq x_{A}$ is equivalent to $\frac{\delta(1-\rho)}{1-\rho^{2} \delta^{2}} \leq$ $x_{A}$. Since $1-\widetilde{m} \leq x_{A}$, it is enough to prove $\frac{\delta(1-\rho)}{1-\rho^{2} \delta^{2}} \leq 1-\widetilde{m}$.

(a) Assume first $\widetilde{m}=\frac{(1-\delta)(1+\rho \delta)}{1-\rho \delta^{2}}$. We have to prove $\frac{\delta(1-\rho)}{1-\rho^{2} \delta^{2}} \leq 1-$ $\frac{(1-\delta)(1+\rho \delta)}{1-\rho \delta^{2}}$, which holds trivially.

(b) Assume now $\widetilde{m}=\frac{1-\rho}{1-\rho^{2} \delta^{2}}$. We have to prove $\frac{\delta(1-\rho)}{1-\rho^{2} \delta^{2}} \leq 1-\frac{1-\rho}{1-\rho^{2} \delta^{2}}$, which holds because $(\rho, \delta) \notin P D$.

B's strategy is optimal when he is the proposer. If $B$ offers $\left(x_{A}, x_{B}\right)$ with $x_{A}>1-\widetilde{m}$, then his offer is accepted, but his final payoff decreases. If $B$ proposes $\left(x_{A}, x_{B}\right)$ with $x_{A}<1-\widetilde{m}$, then his offer is rejected and his final payoff is at most $\rho^{2} \delta^{2} \widetilde{m}+1-\rho$. This deviation is not profitable when $\rho^{2} \delta^{2} \widetilde{m}+1-\rho \leq \widetilde{m}$, i.e. $\frac{1-\rho}{1-\rho^{2} \delta^{2}} \leq \widetilde{m}$, which holds by definition.

B's strategy is optimal when he is the responder. Assume $B$ is the responder and the offer is $\left(x_{A}, x_{B}\right)$. Thus, $B$ 's final payoff after rejection is $\rho \delta \widetilde{m}+(1-\rho) x_{B}$. Perfectness implies that $B$ rejects if $x_{B}<\rho \delta \widetilde{m}+(1-\rho) x_{B}$ and accepts if $x_{B}>\rho \delta \widetilde{m}+(1-\rho) x_{B}$. However, it is equivalent to say that $B$ rejects when $x_{B}<\delta \widetilde{m}$ and accepts when $x_{B}>\delta \widetilde{m}$. This completes the proof that $B$ 's strategy is optimal (when the state is Favor $A$ ).

A's strategy is optimal when she is the proposer. Assume $A$ is the proposer. She may deviate in two possible ways: 1 . by making an acceptable offer (by the transition rule, this means $x_{B} \geq 1-\widetilde{m}$ and thus $x_{A} \leq \widetilde{m}$ ) or 2 . by making an unacceptable offer $\left(x_{B}<1-\widetilde{m}\right)$.

1. The deviation is not profitable when $x_{A} \leq \widetilde{M^{r}}$, and since $x_{A} \leq \widetilde{m}$, it is enough to prove that $\widetilde{m} \leq \widetilde{M^{r}}$, i.e. $\widetilde{m} \leq \rho \delta(1-\widetilde{m})+1-\rho$; equivalently, $\widetilde{m} \leq 1-\frac{\rho}{1+\rho \delta}$. 
(a) Assume first $\widetilde{m}=\frac{(1-\delta)(1+\rho \delta)}{1-\rho \delta^{2}}$. We have to prove $\frac{(1-\delta)(1+\rho \delta)}{1-\rho \delta^{2}} \leq$ $1-\frac{\rho}{1+\rho \delta}$, which holds because $(\rho, \delta) \notin I A$.

(b) Assume now $\widetilde{m}=\frac{1-\rho}{1-\rho^{2} \delta^{2}}$. We have to prove $\frac{1-\rho}{1-\rho^{2} \delta^{2}} \leq 1-\frac{\rho}{1+\rho \delta}$, which holds because $\delta<1$.

2. The deviation is not profitable when $\rho^{2} \delta^{2} \widetilde{m}+(1-\rho) x_{A} \leq \widetilde{M^{r}}$.

Since $x_{A} \leq 1$, it is enough to prove that $\rho^{2} \delta^{2} \widetilde{m}+1-\rho \leq \widetilde{M^{r}}$, i.e. $\rho^{2} \delta^{2} \widetilde{m}+1-\rho \leq \rho \delta(1-\widetilde{m})+1-\rho$; equivalently, $\widetilde{m} \leq \frac{1}{1+\rho \delta}$.

(a) Assume first $\widetilde{m}=\frac{(1-\delta)(1+\rho \delta)}{1-\rho \delta^{2}}$. We have to prove $\frac{(1-\delta)(1+\rho \delta)}{1-\rho \delta^{2}} \leq \frac{1}{1+\rho \delta}$, i.e. $1-2(1-\rho) \leq \rho^{2} \delta^{2}+\rho \delta(1-\rho)$; since $(1-\rho)^{2}>0$, it is enough to prove that $1-2(1-\rho)+(1-\rho)^{2} \leq \rho^{2} \delta^{2}+\rho \delta(1-\rho)$, i.e. $\rho^{2} \leq \rho^{2} \delta^{2}+\rho \delta(1-\rho)$, which holds because $(\rho, \delta) \notin I A$.

(b) Assume now $\widetilde{m}=\frac{1-\rho}{1-\rho^{2} \delta^{2}}$. We have to prove $\frac{1-\rho}{1-\rho^{2} \delta^{2}} \leq \frac{1}{1+\rho \delta}$, which holds trivially.

Agreement: Assume now we are in Agreement. Following these strategies, $A$ proposes $(u, 1-u)$ and $B$ accepts if $1-u>\delta \widetilde{m}$. Thus, the proposal is accepted when $u<\widetilde{M^{a}}$.

1. If $A$ changes her strategy and proposes an unacceptable offer (i.e. $\left(x_{A}, x_{B}\right)$ with $\left.x_{B}<1-\widetilde{m}\right)$, she gets no more than $\rho^{2} \delta^{2} \widetilde{m}+(1-\rho) x_{A}$. Thus, we need to prove that $\rho^{2} \delta^{2} \widetilde{m}+(1-\rho) x_{A} \leq u$. Since $x_{A} \leq 1$ and $\widetilde{m} \leq u$, it is enough to prove that $\rho^{2} \delta^{2} \widetilde{m}+1-\rho \leq \widetilde{m}$, i.e. $\frac{1-\rho}{1-\rho^{2} \delta^{2}} \leq \widetilde{m}$, which holds by definition.

2. If $A$ changes her strategy and proposes an acceptable offer (i.e. $\left(x_{A}, x_{B}\right)$ with $\left.x_{B} \geq 1-\widetilde{m}\right)$, she gets no more than $\widetilde{m}$. Since $u \geq \widetilde{m}$, this deviation is not profitable.

Hence, $\left[\widetilde{m}, \widetilde{M^{a}}\left[\subset U^{a}\right.\right.$. By a slight change of $B$ 's strategy in Favor $A$ when he is the responder, it is analogous to check that $\widetilde{M^{a}} \in U^{a}$. Hence, $\left[\widetilde{m}, \widetilde{M^{a}}\right] \subset U^{a}$.

Delay: Assume now we are in Delay. Following these strategies, $A$ proposes $\left(\frac{u-\rho \delta(1-\widetilde{m})}{1-\rho}, 0\right)$, and $B$ rejects the offer. The final payoff for $A$ is 
$\rho \delta(1-\widetilde{m})+(1-\rho) \frac{u-\rho \delta(1-\widetilde{m})}{1-\rho}=u$. By an analogous reasoning as in Agreement, we deduce that no deviation is profitable as long as $u \in\left[\widetilde{m}, \widetilde{M^{r}}\right]$. Hence, $\left[\widetilde{m}, \widetilde{M^{r}}\right] \subset U^{r}$.

We have then $\left[\widetilde{m}, \widetilde{M^{a}}\right] \subset U^{a}$ and $\left[\widetilde{m}, \widetilde{M^{r}}\right] \subset U^{r}$, from where we conclude that $\left[\widetilde{m}, \widetilde{M^{r}}\right] \subset U$.

We now prove that $u \in\left[\widetilde{m}, \widetilde{M^{r}}\right]$ for all $u \in U$.

Let $M^{a}$ be the supremum of the SP equilibrium payoffs in which the proposer's initial offer is accepted. Let $M^{r}$ be the supremum of the SP equilibrium payoffs in which the proposer's initial offer is rejected. Clearly, $M=\max \left\{M^{a}, M^{r}\right\}$. Notice that both $M^{a}$ and $M^{r}$ are well-defined because $U^{a}$ and $U^{r}$ are nonempty.

Claim $5 M^{a} \leq \min \left\{1-\delta+\delta^{2} M, 1-\frac{\delta(1-\rho)}{1-\rho^{2} \delta^{2}}\right\}$.

Proof. Assume we are in a SP equilibrium in which the first offer of the proposer, say $A$, is accepted. Thus, $A$ proposes $\left(x_{A}, x_{B}\right)$ with $x_{A}=u \in U^{a}$ and $B$ agrees. If $B$ disagreed, then he would get at least $\rho \delta m+(1-\rho) x_{B}$. Thus, the fact that $B$ agrees with $x_{B}$ implies that $\rho \delta m+(1-\rho) x_{B} \leq x_{B}$. This means that $\delta m \leq x_{B}$. Since $u \leq 1-x_{B}$, we deduce $M^{a} \leq 1-\delta m$. This implies (with Lemma 1) $M^{a} \leq 1-\delta(1-\delta M)=1-\delta+\delta^{2} M$ and (under(1)), $M^{a} \leq 1-\frac{\delta(1-\rho)}{1-\rho^{2} \delta^{2}}$.

Claim $6 M^{r} \leq \min \left\{\rho \delta^{2} M+1-\rho, \rho^{2} \delta \frac{1-\rho \delta^{2}}{1-\rho^{2} \delta^{2}}+1-\rho\right\}$.

Proof. Assume we are in a SP equilibrium in which the offer of the proposer, say $A$, is rejected. Thus, $A$ proposes $\left(x_{A}, x_{B}\right)$ and $B$ rejects the offer. Let $u \in U^{r}$ be $A$ 's final payoff. Thus, $u=\rho \delta v+(1-\rho) x_{A}$ with $v \leq 1-u^{\prime}$ such that $u^{\prime} \in U$. Since $1-u^{\prime} \leq 1-m$ and $x_{A} \leq 1$, we have $u \leq \rho \delta(1-m)+1-\rho$ and thus $M^{r} \leq \rho \delta(1-m)+1-\rho$. This inequality implies (with Lemma 1), $M^{r} \leq \rho \delta^{2} M+1-\rho$ and (under (1)),

$$
M^{r} \leq \rho \delta\left(1-\frac{1-\rho}{1-\rho^{2} \delta^{2}}\right)+1-\rho=\rho^{2} \delta \frac{1-\rho \delta^{2}}{1-\rho^{2} \delta^{2}}+1-\rho .
$$

We use Claim 5 and Claim 6 in order to prove $M=\widetilde{M}^{r}$. We have two cases: 
Case 1. $M=M^{a}$.

Subcase 1a. $\widetilde{m}=\frac{(1-\delta)(1+\rho \delta)}{1-\rho \delta^{2}}$. Under Claim $5, M \leq 1-\delta+\delta^{2} M$; equivalently, $M \leq \frac{1}{1+\delta}$. Thus, it is enough to prove $\frac{1}{1+\delta} \leq \widetilde{M^{r}}=\rho \delta(1-\widetilde{m})+$ $1-\rho$, i.e. $\frac{1}{1+\delta} \leq \rho \delta\left(1-\frac{(1-\delta)(1+\rho \delta)}{1-\rho \delta^{2}}\right)+1-\rho$, which holds because $(\rho, \delta) \notin I A$.

Subcase 1b. $\widetilde{m}=\frac{1-\rho}{1-\rho^{2} \delta^{2}}$. Under Claim $5, M \leq 1-\frac{\delta(1-\rho)}{1-\rho^{2} \delta^{2}}$. Thus, it is enough to prove $1-\frac{\delta(1-\rho)}{1-\rho^{2} \delta^{2}} \leq \widetilde{M^{r}}=\rho \delta(1-\widetilde{m})+1-\rho$, i.e. $\rho-\delta+\rho \delta \leq$ $\rho^{3} \delta^{2}+\rho^{2} \delta-\rho^{3} \delta^{3}$. Since $\widetilde{m}=\max \left\{\frac{(1-\delta)(1+\rho \delta)}{1-\rho \delta^{2}}, \frac{1-\rho}{1-\rho^{2} \delta^{2}}\right\}$, we have $\frac{(1-\delta)(1+\rho \delta)}{1-\rho \delta^{2}} \leq \frac{1-\rho}{1-\rho^{2} \delta^{2}}$; equivalently, $\rho-\delta+\rho \delta \leq 2 \rho^{2} \delta^{2}+\rho^{3} \delta^{3}-\rho^{2} \delta^{3}-$ $\rho^{3} \delta^{4}$. Thus, it is enough to prove $2 \rho^{2} \delta^{2}+\rho^{3} \delta^{3}-\rho^{2} \delta^{3}-\rho^{3} \delta^{4} \leq$ $\rho^{3} \delta^{2}+\rho^{2} \delta-\rho^{3} \delta^{3}$, i.e. $-\rho^{3} \delta^{2}(1-\delta)^{2} \leq \rho^{2} \delta(1-\delta)^{2}$, which holds trivially.

Case 2. $M=M^{r}$.

Subcase 2a. $\widetilde{m}=\frac{(1-\delta)(1+\rho \delta)}{1-\rho \delta^{2}}$. Under Claim $6, M \leq \rho \delta^{2} M+1-\rho$; equivalently, $M \leq \frac{1-\rho}{1-\rho \delta^{2}}$. Thus, it is enough to prove $\frac{1-\rho}{1-\rho \delta^{2}} \leq \widetilde{M^{r}}=\rho \delta(1-\widetilde{m})+$ $1-\rho=\rho \delta\left(1-\frac{(1-\delta)(1+\rho \delta)}{1-\rho \delta^{2}}\right)+1-\rho$; i.e. $\frac{1-\rho}{1-\rho \delta^{2}} \leq \frac{\rho \delta^{2}(1-\rho)}{1-\rho \delta^{2}}+1-\rho$, which holds trivially.

Subcase 2b. $\widetilde{m}=\frac{1-\rho}{1-\rho^{2} \delta^{2}}$. Under Claim 6, $M \leq \rho^{2} \delta \frac{1-\rho \delta^{2}}{1-\rho^{2} \delta^{2}}+1-\rho$. Thus, it is enough to prove $\rho^{2} \delta \frac{1-\rho \delta^{2}}{1-\rho^{2} \delta^{2}}+1-\rho \leq \widetilde{M^{r}}=\rho \delta(1-\widetilde{m})+1-\rho=$ $\rho \delta\left(1-\frac{1-\rho}{1-\rho^{2} \delta^{2}}\right)+1-\rho$, which holds trivially.

This proves that $M=\widetilde{M^{r}}$. We now check that $m=\widetilde{m}$. Assume first $\widetilde{m}=\frac{(1-\delta)(1+\rho \delta)}{1-\rho \delta^{2}}$. Under Lemma 1 ,

$$
\begin{aligned}
m & \geq 1-\delta M=1-\delta \widetilde{M^{r}} \\
& =1-\delta(\rho \delta(1-\widetilde{m})+1-\rho) \\
& =1-\delta\left(\rho \delta^{2} \frac{1-\rho}{1-\rho \delta^{2}}+1-\rho\right) \\
& =\frac{(1-\delta)(1+\rho \delta)}{1-\rho \delta^{2}}=\widetilde{m} .
\end{aligned}
$$


Assume now $\widetilde{m}=\frac{1-\rho}{1-\rho^{2} \delta^{2}}$. Under (1), $m \geq \frac{1-\rho}{1-\rho^{2} \delta^{2}}=\widetilde{m}$. Hence, $U=$ $\left[\widetilde{m}, \widetilde{M^{r}}\right]$.

The last step is to prove that $M^{a}=\widetilde{M^{a}}$ and $m^{r}=m^{a}=m$, where $m^{r}$ (resp. $m^{a}$ ) is the minimum of the SP equilibrium payoffs in which the proposer's initial offer is rejected (resp. accepted).

Since $\left[\widetilde{m}, \widetilde{M^{a}}\right] \subset U^{a}, m^{a} \leq \widetilde{m}=m$. Thus, $m^{a}=m$.

Since $\left[\widetilde{m}, \widetilde{M}^{r}\right] \subset U^{r}, m^{r} \leq \widetilde{m}=m$. Thus, $m^{r}=m$.

Since $\left[\widetilde{m}, \widetilde{M^{a}}\right] \subset U^{a}, \widetilde{M^{a}} \leq M^{a}$. Assume first $\widetilde{m}=\frac{(1-\delta)(1+\rho \delta)}{1-\rho \delta^{2}}$. Under Claim $5, M^{a} \leq 1-\delta+\delta^{2} M ;$ since $M=\rho \delta(1-\widetilde{m})+1-\rho$,

$$
\begin{aligned}
M^{a} & \leq 1-\delta+\delta^{2}(\rho \delta(1-\tilde{m})+1-\rho) \\
& =1-\delta+\delta^{2}\left(\rho \delta^{2} \frac{1-\rho}{1-\rho \delta^{2}}+1-\rho\right) \\
& =1-\delta \frac{(1-\delta)(1+\rho \delta)}{1-\rho \delta^{2}} \\
& =1-\delta \widetilde{m}=\widetilde{M^{a}} .
\end{aligned}
$$

Assume now $\widetilde{m}=\frac{1-\rho}{1-\rho^{2} \delta^{2}}$. Under Claim $5, M^{a} \leq 1-\frac{\delta(1-\rho)}{1-\rho^{2} \delta^{2}}=1-\delta \widetilde{m}=\widetilde{M^{a}}$. Thus, $M^{a}=\widetilde{M^{a}}$.

\section{References}

[1] Avery C. and Zemsky P.B. (1994) Money burning and multiple equilibria in bargaining. Games and Economic Behavior 7, 154-168.

[2] Binmore K.G., Rubinstein A. and Wolinsky A. (1986) The Nash bargaining solution in economic modelling. Rand Journal of Economics 17, 176-188.

[3] Compte O. and Jehiel P. (1999) When outside options force concessions to be gradual. Mimeo.

[4] Compte O. and Jehiel P. (2002) On the role of outside options in bargaining with obstinate parties. Econometrica 70(4), 1477-1517.

[5] Corominas-Bosch M. (2004) Bargaining in a network of buyers and sellers. Journal of Economic Theory 115(1), 35-77. 
[6] Feinberg Y. and Skrzypacz A. (2005) Uncertainty about uncertainty and delay in bargaining. Econometrica 73(1), 69-91.

[7] Fershtman C. and Seidmann D.J. (1993) Deadline effects and inefficient delay in bargaining with endogenous commitment. Journal of Economic Theory 60(2), 306-321.

[8] Harsanyi J.C. (1956) Approaches to the bargaining problem before and after the theory of games: a critical discussion of Zeuthen's, Hick's, and Nash's theories. Econometrica 24, 144-157.

[9] Hart O. (1989) Bargaining and strikes. Quarterly Journal of Economics 104, 25-43.

[10] Houba H. and Bolt W. (2002) Credible threats in negotiations: A game theory approach. Kluwer Academic Publishers.

[11] Jehiel P. and Moldovanu B. (1995) Negative externalities may cause delay in negotiation. Econometrica 63(6), 1321-1335.

[12] Luce R.D. and Raiffa H. (1957) Games and Decisions. John Wiley \& Sons, Inc. New York, London, Sydney.

[13] Ma C.A. and Manove M. (1993) Bargaining with deadlines and imperfect player control. Econometrica 61, 1313-1339.

[14] Manzini P. (1999) Strategic bargaining with destructive power. Economic Letters 65(3), 315-322.

[15] Manzini P. and Mariotti M. (2004) Going alone together: joint outside options in bilateral negotiations. The Economic Journal 114(October), 943-960.

[16] Mauleon A. and Vannetelbosch V.J. (2004) Bargaining with endogenous deadlines. Journal of Economic Behavior \& Organization 54, 321-335.

[17] Muthoo A. (1999) Bargaining theory with applications. Cambridge University Press.

[18] Osborne M.J. and Rubinstein A. (1990) Bargaining and markets. San Diego. Academic Press. 
[19] Raiffa H. (1953) Arbitration schemes for generalized two-person games. Contributions to the Theory of Games II. Ed. by H.W. Kuhn and A.W. Tucker. Princeton, NJ. Princeton University Press.

[20] Rubinstein A. (1982) Perfect equilibrium in a bargaining model. Econometrica 50(1), 97-109.

[21] Selten R. (1975) Reexamination of the perfectness concept for equilibrium points in extensive games. International Journal of Game Theory 4, 2555.

[22] Sjöström T. (1991) Ståhl's bargaining model. Economic Letters 36, 153157.

[23] Zeuthen F. (1930) Problems of monopoly and economic warfare. G. Routledge \& Sons. London. 